\title{
A NEW ADAPTIVE AND SELF ORGANIZING FUZZY POLICY TO ENHANCE THE REAL TIME CONTROL PERFORMANCE.
}

\author{
*RAFIK LASRI, IGNACIO ROJAS, HECTOR POMARES \\ Dep. Computer Architecture \& Computer Technology \\ E.T.S.Ing. Informática \& Telecomunicación - Univ. of Granada \\ Periodista Daniel Saucedo Aranda, s/n, E-18071 Granada (SPAIN) \\ E-mail:\{rlasri;irojas;hector\}@ugr.es \\ OLGA VALENZUELA \\ Dep. Applied Mathematics \\ Univ. of Granada, SPAIN \\ E-mail:olgavc@ugr.es
}

Received 17 January 2013

Accepted 26 September 2013

\begin{abstract}
In this paper, a temperature control in real time control process was presented using several control algorithms. A quantitative comparison based on the real power consumption and (the precision and the robustness) of these controllers during the same control process and under the same conditions will be done. The proposed Adaptive and Self Organizing Fuzzy policy has been able to prove its superiority against the remaining controllers. The new Adaptive and Self Organizing Fuzzy Logic Controller starts the control with a very limited information about the controlled process (delay and the monotonicity sign) and without any kind of offline pre-training, the adaptive controller acts online to collect the necessary background to adapt their rules consequents and to self organize their membership functions from the real behavior of the controlled process. During 200 minutes and under the same conditions all the performed controllers have been used to control the room temperature, each simulation has been repeated five times with two different sets of set points. These amounts of results was used as a set of sampling for the statistical tool ANOVA (Analysis of Variance) that can prove and illustrate the validity and the extrapolability of the conclusions extracted from several stages of this work.
\end{abstract}

Keywords: Adaptive fuzzy policy, self-organize fuzzy controller, real time control, saving power.

\section{Introduction}

In the last decade, the area of intelligent control underwent significant progress by developing new control techniques $[1,2]$ or by modifying the methods previously used [3]. Fuzzy logic controllers have been considered as part of the most used control strategies that attract the attention of researchers in this field. Since, from its first appearance in 1974 by Mamdani

\footnotetext{
* Corresponding Author: Rafik Lasri, Dep. Computer Architecture \& Computer Technology. E.T.S.Ing. Informática \& Telecomunicación. Univ of Granada. Periodista Daniel Saucedo Aranda, s/n, E-18071 Granada (SPAIN). E-mail: rlasri@ugr.es
}

[4], several models of fuzzy controllers which adopt different methodologies and utilize wide range of intelligent techniques that can ensure robust and reliable control have been developed. In the last ten years, Adaptive and Self-Organizing Fuzzy Controllers have been well documented and great improvements regarding the error reduction have been achieved [5]. In fact, this contribution is not limited only in the error reduction but it offers novel prospects for the control process. In other words, this paper presents a new intelligent control methodology based on fuzzy logic control able to adapt and to self-organize their internal parameters in real time; this methodology can guarantee significant improvements in control performance that 
exceeds the traditional performance of conventional controllers (more precision and low power consumption).

The first model of a Self-Organizing Fuzzy Logic Controller (SOFLC) was developed in 1979 [6], the essential idea of this latter was based on the identification of the responsible rule of the poor state of the plant and substitute it with an appropriate value that can minimize the committed error. The SOFLC oftentimes uses only the monotonicity sign to express this reliance (the Jacobian matrix is not always available). Generally, SOFLC systems have demonstrated their usefulness in applications that do not present high level of complexity, since it does not require more than tuning the consequents of the rules. On the other hand, more complex systems require designing more sophisticated controllers, that's because, SOFLCs are only capable of a coarse tuning of the controller parameters [7-11].

Unlike most control systems that appear in the bibliography, the objective of our Adaptive and SelfOrganizing Fuzzy Controller presented in this paper is not limited only in reducing the error committed during the control process, but the proposed methodology can simultaneously ensure a very precise control with a very moderate power consumption. In the area of control process, the power consumption has not been well discussed, since, in the last ten years a limited number of works have been addressed to this issue [1216]. The control systems able to ensure some level of saving power that appear in the bibliography propose two control methods to ensure the reduction of energy consumption. In the first one, the controller is used as a manager of the system that optimizes the use of the energy sources and control the time of use of the actuators to avoid the over-spending [15], while in the second method, the controller optimizes the necessary energy used to feed the actuator of the system via a very moderate control signal [14]. In [16] a comparative study between a static Fuzzy Controller and a set of PID controllers was presented. The fuzzy controller feeds the actuator directly; the obtained results demonstrate his capability of carrying out the control process consuming less energy than the remaining controllers.

In this piece of work, a quantitative comparison of the obtained results using the adaptive controller and a set of conventional controllers under the same conditions will be presented to emphasize the great contribution that can bring the new fuzzy control methodology proposed regarding the reduction of both: the committed error and the electrical cost.

\section{The experimental environment}

The behavior of the controllers designed in this paper will be tested using the same control process and under the same conditions. To do this, a scale model of a room who can imitate the control of temperature of a room has been used (Fig.1). The aim of the control process is to fix the room temperature to predefined values. The temperature control is done by controlling a fan that cools the environment inside the room, while the source of the temperature located in the center of the room tries to heat up the environment.

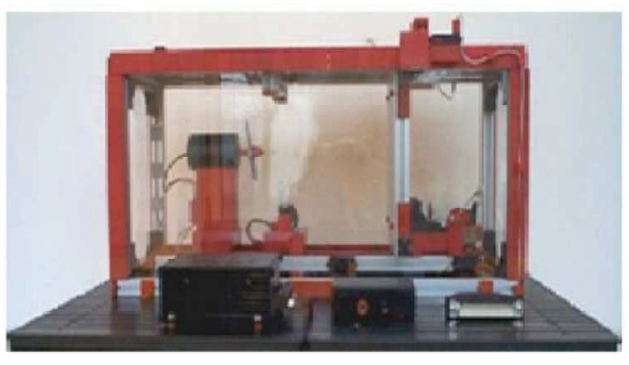

Fig.1. Scale model of a room.

\section{Implementation of the used controllers}

In this section we design the controllers used in this paper. The structure of the controllers used consists of two inputs and one output (MISO). The inputs are the temperature error and its derivative $\left(\boldsymbol{T}_{\boldsymbol{e}}, \boldsymbol{T}_{\dot{\boldsymbol{e}}}\right)$. Since the error is expressed as: $\boldsymbol{T}_{\boldsymbol{e}}=\boldsymbol{T}_{\boldsymbol{s} \boldsymbol{p}}-\boldsymbol{T}_{\boldsymbol{i}}$. The controller output is the supply voltage of the fan which is responsible of cooling the room.

\subsection{Design of a PID controllers}

The heuristic method of Ziegler-Nichols in closed loop offers a suitable tool to easily tune a PID controller [17]. It is performed by setting the $I$ (Integral) and $D$ (Derivative) gains to zero. The $P$ (Proportional) gain is then increased (from zero) until it reaches the ultimate gain $K u$, at which the output of the control loop oscillates with a constant amplitude. $K u$ and the oscillation period $P u$ are used to set the $P, I$, and $D$ gains depending on the type of the desired controller. Table 1 gave us the predefined formulas to tune the PID controllers. 
Table 1. Formulas for the controller parameters in the Ziegler-Nichols closed loop method.

\begin{tabular}{cccc}
\hline & \multicolumn{3}{c}{ Parameters } \\
\cline { 2 - 4 } Structures & $\mathrm{Kp}$ & $\mathrm{Ti}$ & $\mathrm{Td}$ \\
\hline P controller & $0.5 \mathrm{~K} p_{u}$ & $\infty$ & 0 \\
PI controller & $0.45 \mathrm{~K} p_{u}$ & $P_{u} / 1.2$ & 0 \\
PID controller & $0.6 \mathrm{~K} p_{u}$ & $P_{u} / 2$ & $P_{u} / 8=T_{i} / 4$ \\
\hline
\end{tabular}

\subsection{Design of a classical Fuzzy Logic Controller}

Due to its simplicity and ease of implementation the zero-order Takagi-Sugeno-Kang Fuzzy Controller (TSK-0) has been chosen for this simulation. A set of triangular membership function have been assigned to the inputs (seven for the first input and five for the second one) to cover the whole range of variation. Since the controller is a TSK- 0 , then the output is a set of scalar values. The Fig.2 summarizes the global structure of the classical Fuzzy Logic Controller implemented.
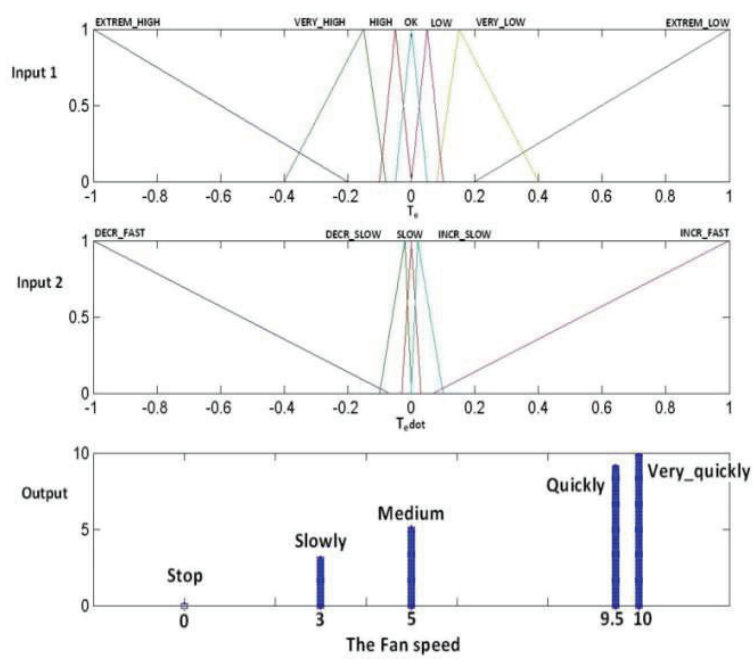

Fig. 2. The Global structure of the classical Fuzzy Logic Controller used in the simulation.

\subsection{Design of Adaptive and Self-Organize Fuzzy Logic Controller}

Basically the mathematical equation of the plant to be controlled can be expressed in terms of its differential equations and/or its difference equations by using a short enough sampling period to obtain that [18]:

$y(k+d)=f(y(k), \ldots, y(k-p), u(k), \ldots, u(k-q))$ where $d$ is the delay of the plant and $f$ is an unknown continuous and differentiable function, $u$ is the plant output and the plant order is determined by the constants $p$ and $q$.

The aim of each control policy is to translate the output to the desired value (within the operation range). The challenge faced with this type of plants is for each input value we must obtain a well known output. Furthermore, the partial derivative of the plant output with respect to the control input must be different from zero for any input within the operation range. As plants are continuous, this means that the mentioned derivative must have a constant sign $[10,19]$.

Using the same structure of the classical FLC before designed (TSK-0 and the same distribution of MFs) we proceed to approximate such function. The complete set of rules of the controller could be presented as:

$$
\begin{gathered}
I F x_{1} \text { is } X_{1}^{i_{1}} A N D x_{2} \text { is } X_{2}^{i_{2}} A N D \ldots x_{N} \text { is } X_{N}^{i_{N}} \\
\text { THEN } u=R_{i_{1} i_{2} \ldots i_{N}}
\end{gathered}
$$

where $\mathrm{X}_{\mathrm{v}}^{\mathrm{i}_{\mathrm{v}}} \in\left\{\mathrm{X}_{1}^{\mathrm{i}_{1}}, \mathrm{X}_{2}^{\mathrm{i}_{2}}, \ldots, \mathrm{X}_{\mathrm{N}}^{\mathrm{i}_{\mathrm{N}}}\right\}$ are the MFs of input $\mathrm{X}_{\mathrm{v}}$, $n_{v}$ is the number of MFs for that input variable and $R_{i_{1} i_{2} \ldots i_{N}}$ is a numerical value representing the rules consequent. For the inference method, we will use the product as T-norm, while the "centre of gravity" with sum-product is chosen as defuzzification strategy. Hence, the fuzzy controller's output may be expressed as:

$$
\hat{F}\left(\vec{x}^{k}\right)=\frac{\sum_{i_{1}=1}^{n_{1}} \sum_{i_{2}=1}^{n_{2}} \cdots \sum_{i_{N}=1}^{n_{N}}\left(R_{i_{1} i_{2} \cdot i_{N}} \cdot \prod_{v=1}^{N} u_{x_{v}^{i_{v}}}\left(x_{v}^{k}\right)\right)}{\sum_{i_{1}=1}^{n_{1}} \sum_{i_{2}=1}^{n_{2}} \cdots \sum_{i_{N}=1}^{n_{N}}\left(\prod_{v=1}^{N} u_{x_{v}^{i_{v}}}\left(x_{v}^{k}\right)\right)}
$$

where $\overrightarrow{\mathrm{x}}^{\mathrm{k}}$ is the $\mathrm{N}$-dimensional input vector at instant $\mathrm{k}$. The objective of the proposed new methodology is to design an adaptive control algorithm able to adapt and to self-organize their internal parameters in real time using only some qualitative information about the plant. The main features of this novel methodology are the following:

- No need any mathematical modeling and don't require any kind of differential equations of the controlled plant.

- The algorithm doesn't need prior information about the control strategy. The new adaptive fuzzy controller can start the control process without allocate a rule base or by assigning arbitrary values.

- The fuzzy system does not use specific information about the dynamic of the plant. Therefore, its behavior does not rely on it and the 
adaptive controller can compensate any kind of unexpected conditions, being that the adaptive ability of the proposed methodology can learn how to turn back the controlled variable within the allowable range.

At the first stage of the algorithm we proceed to adapt the consequents of the rules as a reward/penalty, this process can guarantee a significant reduction of the error committed during the control process. In the second stage, the process of optimizing the membership functions begins with the aim to balance the inputs space regarding to the contribution of the accumulated error registered during the previous training period. This fine tuning can contribute in improving the control performance. Since, the new antecedents of rules will be more appropriate for the current state of the plant. Fig.3 (A) presents the closed-loop control system with the proposed control algorithm.

\subsubsection{Stage of Auto-learning: Adaptation of the consequents of the rules}

The mean goal of our approach is to ensure a high level of accuracy and stability of the control process in real time. So for that, we should equip our fuzzy controller with a suitable skill that can make the control algorithm able to "learn" which actions lead to the desired set point. At this stage, the learning process is used to correct the consequents of the fuzzy rules and these latter will be used to provide the new "improved" results.
However, the modification of the controller's parameters is considered as a difficult task due to the ignorance of the real internal behavior of the used plant. The use of the gradient-descent algorithm can solve this problem but we would need to handle the partial derivative of the plant output with respect to the control input $(\partial \mathrm{y} / \partial \mathrm{u})$. Therefore, it makes us face two serious difficulties: Firstly, the main objective of our approach is to control a plant that we don't know their differential equation; hence, their derivative will be unknown too.

Secondly, the approximation of the aforementioned derivative by $\Delta y / \Delta u$ is strongly depending to the sampling period i.e., in cases where these sampling periods are relatively large, the approximation cannot be made. However, we are aware that the plant must be under control, if that, its derivative will have a definite constant sign. Therefore, the use of the information of how the monotonicity of the plant change regarding to the control input can indicate us in which direction we have to move the rule consequents to get the required improvement $[5,10]$.

In the proposed methodology, the adaptation mechanism evaluates the plant's state periodically and suggests a suitable correction (either a reward or a penalty) for the rules responsible of reaching such state. In the cases where the monotonicity is positive (i.e., the plant output increases as the control input increases)

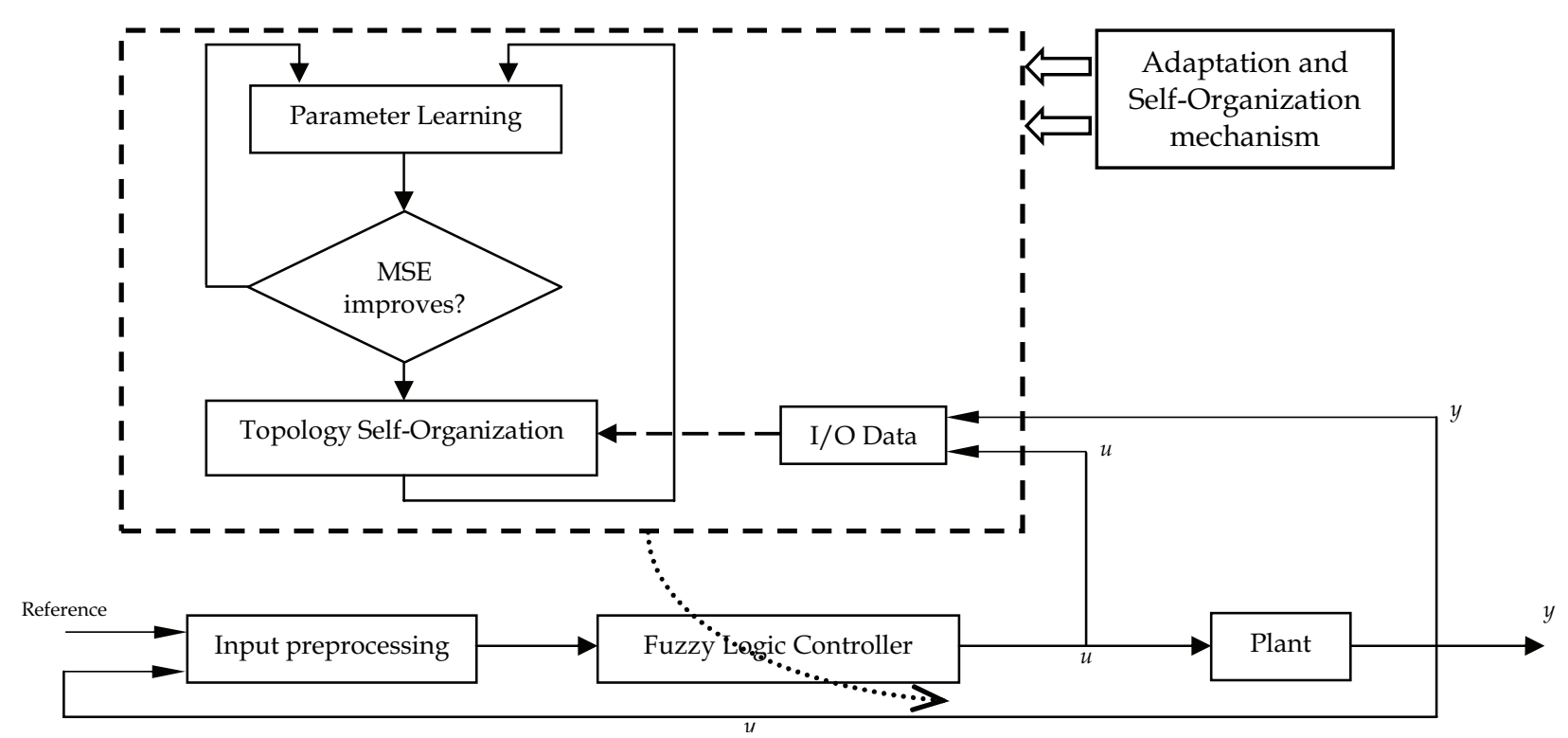

Fig. 3 (A). Representation of the closed-loop control system with the On-Line Adaptive and Self-Organizing Fuzzy Logic. 
and, at the instant $k+1$, the output obtained is bigger than required $(y(k+1)>r(k))$, it means that the control signal used at the instant $k$ should have been lower; similarly, if the obtained output is lower than desired $(y(k+1)<r(k))$, then the control input should have been bigger. Using the same analogy for the case of negative monotonicity, only the direction of the movements needs to be exchanged. It's important to note here that in such state, only some rules will be activated, so the reward/penalty correction must be applied just on the rules responsible of the current state of the plant. Likewise, the proposed correction must be proportional to the degree of activation of the involved rules.

Consequently, in our approach we proceed to modify the consequents of the $i$-th rule of the fuzzy system using the following expression:

$$
\begin{aligned}
& \Delta R_{i_{1} i_{2} \cdots i_{N}}(k)=C \cdot \mu_{i_{1} i_{2 \cdots i_{N}}}(k-d) \cdot e_{y}(k) \\
& =C \cdot \mu_{i_{1} i_{2 \cdots i_{N}}}(k-d) \cdot(r(k-d)-y(k))
\end{aligned}
$$

where $d$ is the time delay, $\mu_{i}(k-d)$ is the activation degree of the $i$-th rule at instant $k$ - $d, r(k-d)$ is the required set point of the plant output at that time and $y(k)$ is the plant output. The $C$ value represents a normalization constant with the same sign as the monotonicity of the plant with respect to $u$ and whose absolute value can be set offline as $|C|=\Delta u / \Delta r$, where $\Delta u$ is the range of the controller's actuator, and $\Delta r$ is the range in which the reference signal varies. These two values are set according to the human expert knowledge. We must note here that in (4) the use of $r(k$ $d)$ instead $r(k)$ is justified, because the rules that are activated at instant $k$ - $d$ serve to achieve the desired value $r(k-d)$ and not $r(k)$.

Simultaneously with these steps, we have to be careful when we run our algorithm in real time, that's because the majority of the real time controllers face some limitations on their operation, which adversely affects the performance of the control process. A simple example of these kinds of limitations is the actuator range, in the cases where this range is limited on $\left[u_{\mathrm{min}}\right.$, $\left.u_{\max }\right]$ and the suitable control input needed to reach the reference value is outside of this range, the controller will provide the extreme limit of the actuator range as optimal value. Hence, at the next instant, the desired set point will not be achieved. However, in similar cases, the rules are optimized and the adequate output is generated. For that, it will be unfair re-proceed to modify these rules. The solution of this undesired state resides in accomplishing the following two conditions before applying the suggested corrections:

$$
\begin{aligned}
& \text { IF } u(k-d)=\mathrm{u}_{\min } \text { AND } \Delta \mathrm{R}_{\mathrm{i}}(k)<0 \text { THEN } \Delta \mathrm{R}_{\mathrm{i}}(k)=0 \\
& \text { IF } u(k-d)=\mathrm{u}_{\max } \text { AND } \Delta \mathrm{R}_{\mathrm{i}}(k)>0 \text { THEN } \Delta \mathrm{R}_{\mathrm{i}}(k)=0
\end{aligned}
$$

At the end of this section, we have to clarify that the proposed adaptation process is running in real time, from the startup of control until the end. Since, the adaptation involves (in real time) important improvements to the control performances.

\subsubsection{Stage of Self-Organization: Optimization of the membership functions}

In the adaptation stage, the rule consequents were adopted to accomplish the best control performance. Based on the knowledge about the system to be controlled, the fuzzy controller uses a fix predefined structure (i.e., number of inputs, number of MFs and their parameters, etc.). Fixing such structure may not be always easy, since, it requires a deep knowledge about the system to be controlled $[5,19]$. Therefore, in order to start the control of the plant using very limited information, we need to use advanced adaptive skills that exceed the aforementioned adaptive process, i.e., more internal parameters of the used controller need to be adapted and optimized. Nevertheless, important number of works that appear in the literature present adaptive controllers with prefixed structure [20, 21, 22], hence, there are some risks that the plant may not be adequately represented [19].

The methodology suggested in this work is based on the use of large amount of data provided from the real behavior of the control process; the Input/output data extracted in real time can reflect important information about the real inverse function of the controlled plant. Thus, in the case where the control input is $u(k)$ at instant $k$, and response of the plant was $y(k+d)$, we can have more important information than just the error of the plant. If in the same state, we get the reference value $r\left(k^{\prime}\right)=y(k+d)$ then the optimal control input will be $u(k)$ independently of $y(k+1)$ is the desired output or not. The aforementioned statement is valid for static systems while in systems where the dynamics change rapidly in time this statement do not hold, since; the previewed data obtained and stored are no longer valid, because the I/O relationships keep changing with the time. In our approach we use this information to set the self 
organizing fuzzy controller; we store the I/O data obtained from the real behavior of the plant in a memory $M$. After that, we use these data to re-organize the emplacement of the MFs. During the operation of the controller all the I/O data will be stored, that mean a very big amount of data that can explode the memory. To overpass this inconvenience, we define the memory $M$ as a fixed grid in the input space. Hence, we will get new datum that will be stored (without assigning any weights to this data) in the corresponding hypercube defined by this grid every time. The fact of no weights are assigned to the data is due to avoid locale treatment of the obtained information. Since, the reference signal in real time control process may stay constant during a certain time. So, in the case where the stored data are all weighted, the weight of every cell would grow making all the information stored in the previous cells irrelevant. Therefore, our approach would be based only on local information. In contrary case, all the data stored in the memory $M$ would be considered as relevant information, and therefore, the data stored in $M$ reflects a uniform representation of the input space of the inverse function of the plant.

Solving functional-approximation problems require full information about the operating regions of the plant. In real time control process, we were able to use just the information obtained during the controller's normal operation. Therefore, we cannot be sure that the normal operation of the system can span the integrity of the input space. In fact, when the control process reaches a certain region, the operation of the system generates the information about this region, which forms a major priority for the controller.
However, we know that to perform a full functional approximation of the inverse function of the plant, an important amount of information is required.

In the proposed methodology, this approximation is feasible in the region of interest. This provides a good control performance in the operating regions, even if the approached inverse function of the plant is not valid in other areas. Although the control does not reach these regions, the proposed mechanism of auto learning will learn how to control them.

In [23], the new incoming training data are used to make a decision about how the topology can be modified, that mean different amount of data may lead to different structures. In a different way, our approach use the integrity of the data stored from the whole operating region of interest in order to establish a new topology for the used controller, this latter can improve significantly the control performances.

The proposed methodology contains two phase (Adaptation and Self Organization). The commutation from the first one to the second one occurs when the improvement caused by the consequents adaptation don't progress anymore.

We note here that to make adequate decisions in the reorganization of the MFs, no topology changes are performed until the amount of data in the memory $M$ is representative, that because we are not sure of the accuracy level of the behavior of the plant in the initial functioning of the controller (in this case, a threshold for the percentage of empty hyper-cubes in the grid is used).

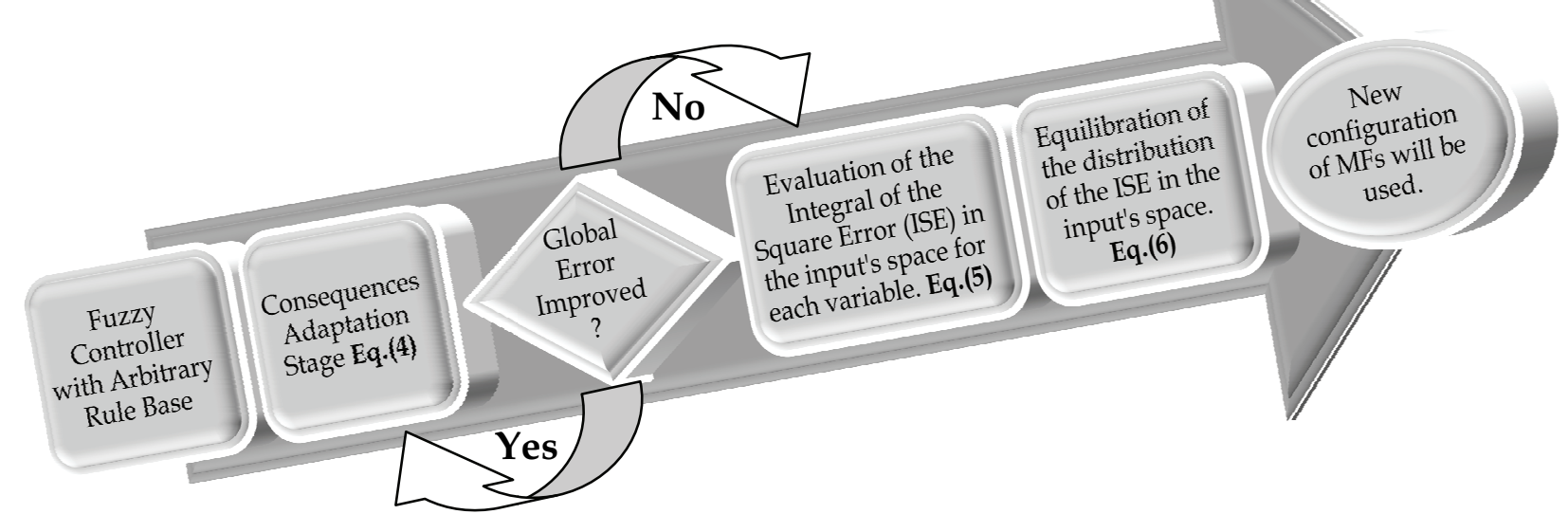

Fig. 3(B). General flowchart of the proposed methodology. 
Fig.3(B) presents the general flowchart of the methodology proposed in this paper. The controller can start the control process with no initial parameters or by using an arbitrary set of rules. Moreover, our adaptive controller operates in real time and the proposed process never finishes.

The re-organization process is based in the idea that each area in the inputs space must have a balanced contribution to the Integral of the Square Error (ISE) registered during the control process. Since, the region that has a big contribution to the ISE is the region more frequently activated. The equation for the ISE is:

$$
\begin{aligned}
I S E_{i}^{j}=\frac{1}{\sigma_{y}^{2}}\left(\int e^{2}(t) d t\right. & \left.\right|_{x_{i}(t) \epsilon\left[c_{i}^{j-1}, c_{i}^{j}\right]} \\
& \left.-\left.\int e^{2}(t) d t\right|_{x_{i}(t) \epsilon\left[c_{i}^{j}, c_{i}^{j+1}\right]}\right)
\end{aligned}
$$

where $\mathrm{c}_{\mathrm{j}}$ presents the position of the $j$-th membership function, $x_{\mathrm{i}}$ is the input and $\sigma_{\mathrm{y}}$ is the variance of the centre of the plant output, is used as a normalization factor. The first integral calculates the error, if the input is between the $(j-1)$ center and the $j$ center. The second integral calculates the error, if the input is between the $j$ center and the $(j+1)$ center. This equation can be simplified for two reasons firstly, the system is discrete which implies that the integral can be written as a sum and secondly, our input is the error of the temperature, so this error does not have to be calculated.

The sign of the estimated ISE determines the displacement direction of the centers of the MFs, since a positive ISE means that the error in the left region is larger than the right one; therefore, the center of the membership function has to be moved to the left and vice-versa. This displacement should not affect the order of the MFs, using the following equation we can guarantee this criterion:

$$
\Delta c^{j}= \begin{cases}\frac{c^{j-1}-c^{j}}{2} \frac{I S E^{j}}{I S E^{j}+\frac{1}{R^{j}}}, & \text { if } I S E^{j} \geq 0 \\ \frac{c^{j+1}-c^{j}}{2} \frac{\left|I S E^{j}\right|}{\left|I S E^{j}\right|+\frac{1}{R^{j}}}, & \text { if } I S E^{j}<0\end{cases}
$$

the parameter $\mathrm{R}^{\mathrm{j}}$ precise the degree of displacement of the centers. An important $\mathrm{R}^{\mathrm{j}}$ leads to an important displacement of the center and a small $\mathrm{R}^{\mathrm{j}}$ produces a small displacement. In this case, the controller starts with high values of $\mathrm{R}$ (e.g., 100) and it will be decreased exponentially every $\mathrm{T}$ time. $\mathrm{T}$ has the size of one learning run. During the first $\mathrm{T}$ the consequences would be tuned and the sum will be calculated simultaneously without estimate the ISE. At the second $\mathrm{T}$, the ISE will be calculated and the new configuration of the membership functions would be generated after the last iteration of the second $T$. This new configuration will be used in the next run (in the third run the controller does not calculate the ISE) to re-tune the consequents output and generate a full optimized output. In fact this process is endless; while the controller operates this task will be repeated periodically but using a smaller value of $\mathrm{R}$.

\section{Results Analysis and Conclusion}

This study aims to illustrate the superiority of the proposed methodology and approve its validity by a statistical analysis of the results obtained from a set of real time simulations. For this, each simulation (200 minutes of control with several set-points) will be repeated five times and with two different sets of setpoints under the same conditions to form a set of sampling, this latter will be used by the statistical tool ANOVA to realize the expected analysis. The criterion of comparison between the different methodologies used are the committed error (MSE) and the Electrical Consumption (EC) during the control process. Table 2 (A) presents the results of all simulations performed during this study giving the MSE and the EC obtained of each algorithm using two sets of set-points.

\section{$>$ Analysis of variance (ANOVA)}

To analyze the variance of the MSE we proceed to construct the ANOVA table that decomposes the MSE and EC variability in contributions due to several factors. We note here that the sum of squares type III has been chosen (by default); the contribution of each factor is measured by avoiding the effects of the remaining factors. The $\mathrm{P}$-value test the statistical significance of each factor, a P-value less than 0.05 implies that this factor has a statistically significant effect on the MSE/EC with a 95\% confidence level, so from the data presented in the ANOVA table we can determine if the effects have a statistically significant effect on the MSE/EC.

\section{> Multiple Range Test}

In the Multiples Range Test a multiples comparison procedure is done to determine which means are 
significantly different from others, the method used to discriminate between the means is the method of Least Significant Difference (LSD) of Fisher. The asterisk adjacent to the pairs indicates that these pairs show statistically significant differences with a $95 \%$ confidence level.
The factor A: SET POINTS presents the effect of using two different sets of set-points on the variables studied (MSE and EC) during the control process. The factor B: CONTROL presents the effect of the control algorithms used on the variables studied (MSE and EC) during the control process.

\subsection{Analysis of the MSE}

Table 2 (A). The MSE obtained during each simulation Set points 1

\begin{tabular}{|c|c|c|c|c|c|c|c|}
\hline \multicolumn{2}{|c|}{} & $\mathbf{1}$ & $\mathbf{2}$ & $\mathbf{3}$ & $\mathbf{4}$ & $\mathbf{5}$ & $\sum$ / 5 \\
\hline \multirow{2}{*}{ PI } & MSE & 1.62 & 1.09 & 1.56 & 1.36 & 1.7 & $\mathbf{1 . 4 6}$ \\
\cline { 2 - 8 } & $\mathbf{E C}$ & 1142 & 968.4 & 1159.9 & 1136.7 & 1158 & $\mathbf{1 1 1 3}$ \\
\hline \multirow{2}{*}{ PD } & $\mathbf{M S E}$ & 1.07 & 1.07 & 1.5 & 1.12 & 1.45 & $\mathbf{1 . 2 5}$ \\
\cline { 2 - 8 } & $\mathbf{E C}$ & 1048.2 & 1022 & 1163.4 & 1024.3 & 1109.3 & $\mathbf{1 0 7 4}$ \\
\hline \multirow{2}{*}{ PID } & $\mathbf{M S E}$ & 1.07 & 1.16 & 1.66 & 1.35 & 1.06 & $\mathbf{1 . 2 6}$ \\
\cline { 2 - 8 } & $\mathbf{E C}$ & 1121,1 & 1120.2 & 1151.9 & 1135.2 & 1133.1 & $\mathbf{1 1 3 2 . 3}$ \\
\hline \multirow{2}{*}{ FLC } & $\mathbf{M S E}$ & 0.92 & 1.03 & 1.49 & 1.31 & 1.05 & $\mathbf{1 . 1 2}$ \\
\cline { 2 - 8 } & $\mathbf{E C}$ & 1159.3 & 1140.3 & 1130.9 & 1139.5 & 1108.7 & $\mathbf{1 1 3 5 . 7}$ \\
\hline \multirow{2}{*}{ Adapt } & $\mathbf{M S E}$ & 1.1 & 0.7 & 0.74 & 0.65 & 0.5 & $\mathbf{0 . 7 4}$ \\
\cline { 2 - 8 } FLC & $\mathbf{E C}$ & 1084.4 & 1088.2 & 1084.3 & 932 & 1082.3 & $\mathbf{1 0 5 4 . 2}$ \\
\hline
\end{tabular}

Table 3 (A). Analysis of Variance for MSE (sum of squares type III).

\begin{tabular}{|c|c|c|c|c|c|}
\hline Source & $\begin{array}{c}\text { Sum of } \\
\text { squares }\end{array}$ & DF & Mean Square & F-Ratio & P-Value \\
\hline PRINCIPAL EFFECTS & & & & & \\
\hline A:SET POINTS & 0,069192 & 1 & 0,069192 & 1,98 & 0,1663 \\
\hline B:CONTROL & $\mathbf{2 , 1 8 8 3 1}$ & $\mathbf{4}$ & $\mathbf{0 , 5 4 7 0 7 8}$ & $\mathbf{1 5 , 6 6}$ & $\mathbf{0 , 0 0 0 0}$ \\
\hline RESIDUAL & 1,53669 & 44 & 0,0349247 & & \\
\hline TOTAL & 3,79419 & 49 & & & \\
\hline
\end{tabular}

\section{Multiple Range Test for MSE of B:CONTROL}

Table 4. (A) Method of 95,0\% LSD for MSE of CONTROL.

\begin{tabular}{|c|c|c|c|c|}
\hline CONTROL & Cases & Mean $\boldsymbol{L S}$ & $\sum \boldsymbol{L S}$ & $\begin{array}{c}\text { Homogeneous } \\
\text { Groups }\end{array}$ \\
\hline Adapt & $\mathbf{1 0}$ & $\mathbf{0 , 8 2 4}$ & $\mathbf{0 , 0 5 9 0 9 7 1}$ & $\mathrm{X}$ \\
\hline FLC & 10 & 1,209 & 0,0590971 & $\mathrm{X}$ \\
\hline PID & 10 & 1,275 & 0,0590971 & $\mathrm{X}$ \\
\hline PD & 10 & 1,291 & 0,0590971 & $\mathrm{XX}$ \\
\hline PI & 10 & 1,453 & 0,0590971 & $\mathrm{X}$ \\
\hline
\end{tabular}

Table 5. (A)

\begin{tabular}{|c|c|c|c|}
\hline Contrast & Sig. & Difference & +/- Limits \\
\hline Adapt - FLC & $*$ & $-0,385$ & 0,168437 \\
\hline Adapt - PID & $*$ & $-0,451$ & 0,168437 \\
\hline Adapt - PD & $*$ & $-0,467$ & 0,168437 \\
\hline Adapt - PI & $*$ & $-0,629$ & 0,168437 \\
\hline \multicolumn{4}{|c}{ * Indicates a significant difference. }
\end{tabular}

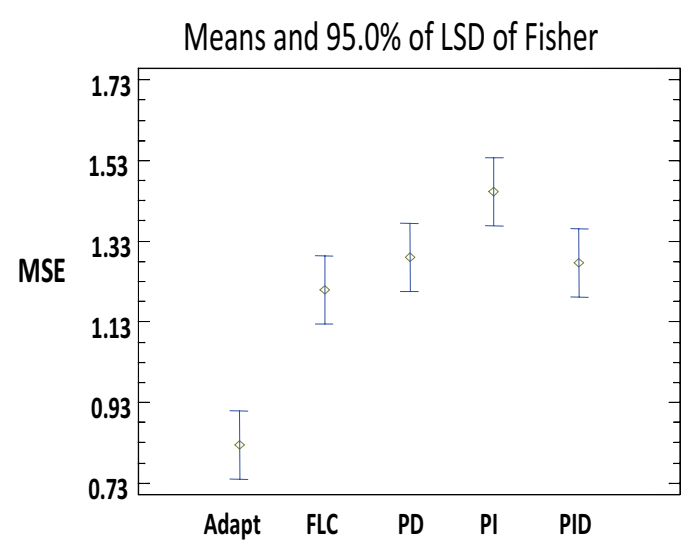

Figure 4. Means of MSE of all used algorithms. 
Table 2 (B). The MSE obtained during each simulation Set points 2

\begin{tabular}{|c|c|c|c|c|c|c|c|}
\hline \multicolumn{2}{|c|}{} & $\mathbf{1}$ & $\mathbf{2}$ & $\mathbf{3}$ & $\mathbf{4}$ & $\mathbf{5}$ & $\sum / \mathbf{5}$ \\
\hline \multirow{2}{*}{ PI } & MSE & 1.22 & 1.28 & 1.35 & 1.7 & 1.65 & $\mathbf{1 . 4 5}$ \\
\cline { 2 - 8 } & EC & 1112 & 1108 & 1106 & 1092 & 1121.3 & $\mathbf{1 1 0 7 . 8}$ \\
\hline \multirow{2}{*}{ PD } & MSE & 1.37 & 1.28 & 1.3 & 1.35 & 1.4 & $\mathbf{1 . 3 5}$ \\
\cline { 2 - 8 } & EC & 1092 & 1120,83 & 1133 & 1089 & 1079.9 & $\mathbf{1 1 0 3}$ \\
\hline \multirow{2}{*}{ PID } & MSE & 1.34 & 1.27 & 1.26 & 1.32 & 1.26 & $\mathbf{1 . 3}$ \\
\cline { 2 - 8 } & EC & 1127.3 & 1102.04 & 1101.1 & 1179.3 & 1121.7 & $\mathbf{1 1 2 6 . 3}$ \\
\hline \multirow{2}{*}{ FLC } & MSE & 1.47 & 1.08 & 1.11 & 1.33 & 1.3 & $\mathbf{1 . 2 5}$ \\
\cline { 2 - 8 } & EC & 1121 & 1098.2 & 1107.4 & 1115.2 & 1162.3 & $\mathbf{1 1 2 0 . 8}$ \\
\hline \multirow{2}{*}{$\begin{array}{c}\text { Adapt } \\
\text { FLC }\end{array}$} & MSE & 1.1 & 1 & 0.83 & 0.78 & 0.84 & $\mathbf{0 . 9}$ \\
\cline { 2 - 8 } & EC & 1187 & 1121.3 & 1009.7 & 1013.8 & 987.5 & $\mathbf{1 0 6 3 . 8}$ \\
\hline
\end{tabular}

Table 3 (B). Analysis of Variance for EC (sum of squares type III).

\begin{tabular}{|c|c|c|c|c|c|}
\hline Source & $\begin{array}{c}\text { Sum of } \\
\text { squares }\end{array}$ & DF & $\begin{array}{c}\text { Mean } \\
\text { Square }\end{array}$ & F-Ratio & P-Value \\
\hline PRINCIPAL EFFECTS & & & & & \\
\hline A:SET POINTS & 85,2035 & 1 & 85,2035 & 0,04 & 0,8504 \\
\hline B:CONTROL & $\mathbf{3 5 3 6 5 , 0}$ & $\mathbf{4}$ & $\mathbf{8 8 4 1 , 2 4}$ & $\mathbf{3 , 7 4}$ & $\mathbf{0 , 0 1 0 6}$ \\
\hline RESIDUAL & 104108, & 44 & 2366,09 & & \\
\hline TOTAL & 139558, & 49 & & & \\
\hline
\end{tabular}

\section{$>$ Multiple Range Test for EC of B: CONTOL}

Table 4. (B) Method of 95,0\% LSD for EC of CONTROL.

\begin{tabular}{|c|c|c|c|c|}
\hline CONTROL & Cases & $\begin{array}{c}\text { Mean } \\
\text { LS }\end{array}$ & $\sum \boldsymbol{L S}$ & $\begin{array}{c}\text { Homogeneous } \\
\text { Groups }\end{array}$ \\
\hline Adapt & $\mathbf{1 0}$ & $\mathbf{1 0 5 9 , 0 5}$ & $\mathbf{1 5 , 3 8 2 1}$ & $\mathrm{X}$ \\
\hline PD & 10 & 1088,19 & 15,3821 & $\mathrm{XX}$ \\
\hline PID & 10 & 1129,29 & 15,3821 & $\mathrm{X}$ \\
\hline FLC & 10 & 1128,28 & 15,3821 & $\mathrm{X}$ \\
\hline PI & 10 & 1110,43 & 15,3821 & $\mathrm{X}$ \\
\hline
\end{tabular}

Table 5. (B)

\begin{tabular}{|c|c|c|c|}
\hline Contrast & Sig. & Difference & +/- Limits \\
\hline Adapt - FLC & $*$ & $-69,23$ & 43,8415 \\
\hline Adapt - PID & $*$ & $-70,244$ & 43,8415 \\
\hline Adapt - PD & & $-29,143$ & 43,8415 \\
\hline Adapt - PI & $*$ & $-51,38$ & 43,8415 \\
\hline \multicolumn{4}{|c|}{$*$ Indicates a significant difference. }
\end{tabular}

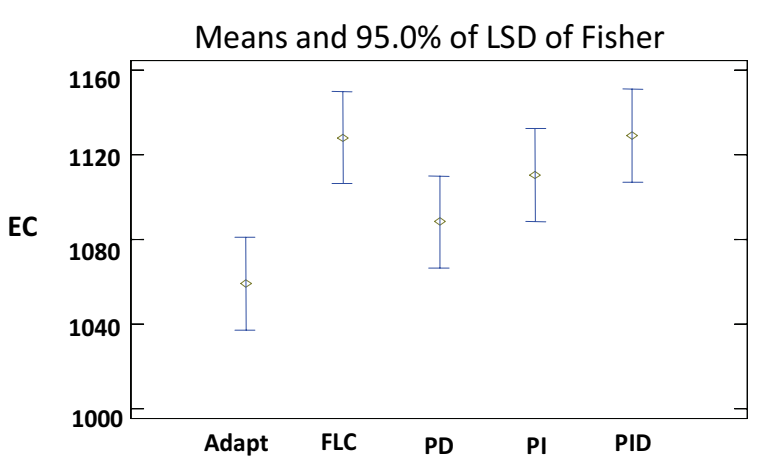

Figure 5. Means of EC of all used algorithms. 


\subsubsection{Analysis of results}

From the ANOVA Tables 3 (A and B) we can deduce that the factor A: SET POINTS does not has a statistically significant effect on the MSE and on the EC registered (P-value $>0.05)$, i.e., the quality of the obtained results in these simulations does not depend on the set-points used. If we change the set-points we will obtain results of the same quality. For the factor B: CONTROL it has a statistically significant effect on the MSE and on the EC registered with a $95 \%$ confidence level (P-value $<0.05$ ), i.e. if we repeat the simulations there is a $95 \%$ risk of having similar MSE and EC during the control process. On the other hand, from the Tables 4 (A), 5 (A) and Tables 4 (B), 5 (B) of Multiples Range test of the MSE and the EC we can deduce that there are significant differences (in MSE and in EC) between our adaptive algorithm and the remaining algorithms with a $95 \%$ level of confidence, i.e. if we repeat the simulations several times there is a $95 \%$ risk of having similar differences of MSE and EC between our algorithm and the others. Also from the same tables, we can observe that when we change the set of set points, the obtained results does not suffer any qualitative changes, i.e., the proposed methodology preserves its superiority against the remaining controllers even when we use different set of set points.

The figures 4 and 5 show clearly the differences between all the used algorithms. In Fig. 4 we can note that the adaptive controller presents the best MSE and in the Fig.5 it's clear that the best EC is registered by the adaptive controller too. However, trough this statistical analysis we have demonstrated that the new Online Adaptive Fuzzy methodology presented in this paper can guarantee several improvements to the control processes that exceed the performance of classical controllers. The extrapolability of the obtained results is clearly emphasized by the use of different set of set-points and by the amount of the simulations performed during this work [1].

\subsection{The perturbation effects on the control performance}

Fig.6 depicts the behavior of the algorithms (static FLC and Adaptive FLC) after a disturbance of $25 \%$ at minute 20. The disturbance used here is a simulation of a temperature decrease caused by a secondary fan and at the 65 minute, we have caused a disturbance of $20 \%$ but this time we simulate a temperature increase caused by another source of temperature. The response of the plant after the disturbances for each algorithm can clearly explain the differences between every one of them. Top of Fig.6 shows the behavior of static FLC in perturbation cases. We can notice how these disturbances can affect the control precision; the static FLC keeps the plant under control but with large error without over passing the perturbation effects.

In the second graph, the adaptive FLC presents a very good performance against these disturbances. It can be clearly noticed that the control precision does not suffer big changes and that the adaptive algorithm can overcome the perturbation effects in few moments later; it can correct the new error and return it within the allowable range. Thus, Table 6 presents a numerical comparison between the error committed in 10 minutes before causing the disturbances and the error committed in 10 minutes with disturbance for each algorithm. This comparison is correct while the 10 minutes used in the calculation of the MSE before and after the perturbation are always from the same set temperature.

Table 6. Comparative performance indices before and after the perturbation

\begin{tabular}{ccc|cc}
\hline & \multicolumn{2}{c|}{$\begin{array}{c}\boldsymbol{\%} 25 \text { in the power of the } \\
\text { Fan }\end{array}$} & \multicolumn{2}{c}{$\begin{array}{c}\text { \% } 20 \text { in the power of } \\
\text { the Fan }\end{array}$} \\
\cline { 2 - 5 } $\begin{array}{c}\text { MSE in 10 } \\
\text { minutes }\end{array}$ & $F L C$ & $\begin{array}{c}\text { Adaptive } \\
F L C\end{array}$ & $F L C$ & $\begin{array}{c}\text { Adaptive } \\
F L C\end{array}$ \\
\hline $\begin{array}{c}\text { Before } \\
\text { perturbation }\end{array}$ & 0.78 & 0.42 & 0.64 & 0.1 \\
\hline $\begin{array}{c}\text { After } \\
\text { perturbation }\end{array}$ & 0.32 & 0.36 & 1.77 & 0.14 \\
\hline
\end{tabular}
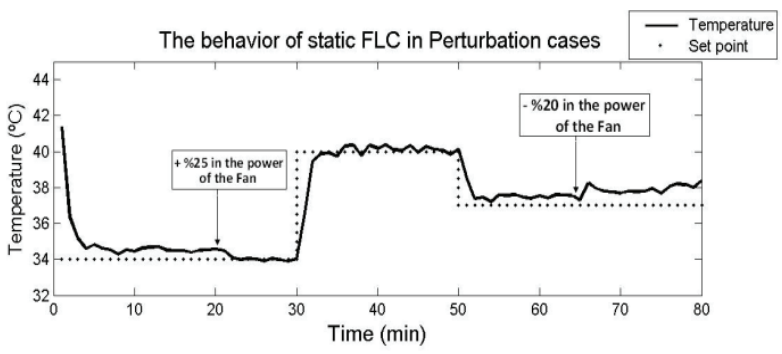

The behavior of Adaptive FLC in Perturbation cases

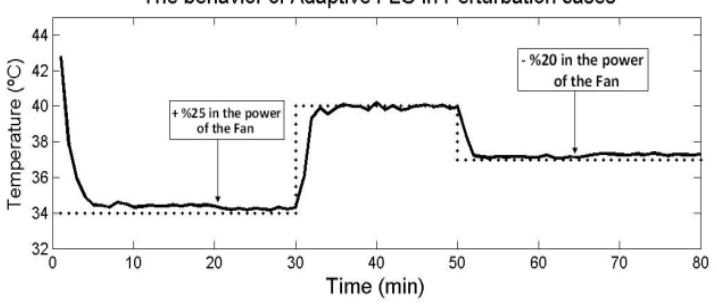

Fig. 6. The perturbation effects on the control performance. 
It is important to clear here that the error reduction in the first case of perturbation doesn't mean a control improvement, it's due to the new Fan added by simulation (+\%25 in the power of the Fan), adding a Fan with power more bigger signify big reduction in the error committed and maybe the error will change the sign if this Fan power was too much. The MSE presented in this table proves that the perturbation effects on the behavior of the adaptive algorithm are very small when compared with their effects on the behavior of the FLC algorithm. The adaptive FLC can quickly compensate the new environmental variations by maintaining the error within the allowable range, whereas the FLC cannot adjust to the new environmental variations. Therefore, the control precision suffers serious changes.

\subsubsection{Statistical analysis of the perturbation results}

After the realization of several test of the behavior of our proposed controller under different types of disturbances, we proceed to approve the validity and the extrapolability of the obtained results using the same analytical tools as in the previous section. To do that (in the case of disturbance) we will use the same types of perturbations (additional Fun and/or source of temperature) but this time with various levels $(+30 \%$, $+15 \%$ and $-30 \%,-15 \%)$ and we will repeat each simulation ( 80 minutes of control) five times using two different sets of set points to form a set of sampling for the statistical tool ANOVA.

Table 7. The MSE obtained under several types of perturbations.

\begin{tabular}{|c|c|c|c|c|c|c|c|c|c|c|c|c|c|}
\hline \multirow{2}{*}{\multicolumn{2}{|c|}{ Perturbation }} & \multicolumn{6}{|c|}{ SET POINTS 1} & \multicolumn{6}{|c|}{ SET POINTS 2} \\
\hline & & \multirow{2}{*}{$\begin{array}{c}\begin{array}{c}\text { MSE } \\
\mathbf{1}\end{array} \\
1.02\end{array}$} & \multirow{2}{*}{$\begin{array}{c}\text { MSE } \\
\mathbf{2} \\
0.82\end{array}$} & \multirow{2}{*}{$\begin{array}{c}\text { MSE } \\
\mathbf{3} \\
0.75\end{array}$} & \multirow{2}{*}{$\begin{array}{c}\text { MSE } \\
\mathbf{4} \\
0.66\end{array}$} & \multirow{2}{*}{$\begin{array}{c}\begin{array}{c}\text { MSE } \\
\mathbf{5}\end{array} \\
0.75\end{array}$} & \multirow{2}{*}{$\begin{array}{c}\sum \mathrm{MSE} / 5 \\
\mathbf{0 . 8}\end{array}$} & \multirow{2}{*}{\begin{tabular}{|c|}
$\begin{array}{c}\text { MSE } \\
\mathbf{1}\end{array}$ \\
1.34 \\
\end{tabular}} & \multirow{2}{*}{$\begin{array}{c}\mathbf{2} E \\
1.03\end{array}$} & \multirow{2}{*}{$\begin{array}{c}\begin{array}{c}\text { MSE } \\
\mathbf{3}\end{array} \\
0.82\end{array}$} & \multirow{2}{*}{$\begin{array}{c}\begin{array}{c}\text { MSE } \\
\mathbf{4}\end{array} \\
1.02\end{array}$} & \multirow{2}{*}{$\begin{array}{c}\begin{array}{c}\text { MSE } \\
\mathbf{5}\end{array} \\
0.8\end{array}$} & \multirow{2}{*}{$\frac{\sum \text { MSE / } 5}{1}$} \\
\hline & Adap FLC & & & & & & & & & & & & \\
\hline & FLC & 1.24 & 1.04 & 1.4 & 1.3 & 1.1 & 1.21 & 1.56 & 1.47 & 1.5 & 1.55 & 1.85 & 1.6 \\
\hline \multirow{2}{*}{$+15 \%$} & Adap FLC & 1.18 & 1.03 & 0.78 & 0.93 & 0.78 & 0.94 & 0.95 & 0.93 & 0.95 & 0.92 & 0.81 & 0.91 \\
\hline & FLC & 1.2 & 1.16 & 1.1 & 1.23 & 1.28 & 1.2 & 1.13 & 1.28 & 1.36 & 1.37 & 1.41 & 1.31 \\
\hline \multirow{2}{*}{$-15 \%$} & Adap FLC & 0,92 & 0.91 & 0.97 & 0.94 & 0.9 & 0.92 & 1.05 & 1.07 & 1.07 & 0.95 & 0.98 & 1 \\
\hline & FLC & 1,3 & 1.16 & 1.2 & 1.17 & 1.5 & 1.26 & 1.1 & 1.14 & 1.27 & 1.58 & 1.86 & 1.4 \\
\hline \multirow{2}{*}{$-30 \%$} & Adap FLC & 1.01 & 0.96 & 0.91 & 0.87 & 0.84 & 0.91 & 1.01 & 0.89 & 1.07 & 0.9 & 0.94 & 0.96 \\
\hline & FLC & 3.76 & 2.96 & 3.72 & 3.88 & 2.7 & 3.4 & 1.84 & 1.9 & 1.66 & 1.7 & 1.26 & 1.7 \\
\hline
\end{tabular}

Table 8. Analysis of Variance for MSE (sum of squares type III).

\begin{tabular}{|c|c|c|c|c|c|}
\hline Source & $\begin{array}{c}\text { Sum of } \\
\text { squares }\end{array}$ & DF & $\begin{array}{c}\text { Mean } \\
\text { Square }\end{array}$ & F-Ratio & P-Value \\
\hline PRINCIPAL EFFECTS & & & & & \\
\hline A:SET POINTS & 0,20402 & 1 & 0,2040 & 0,95 & 0,3341 \\
\hline B:CONTROL & 9,63272 & 1 & 9,6327 & 44,63 & $\mathbf{0 , 0 0 0 0}$ \\
\hline C:NOISE & 5,60313 & 3 & 1,8677 & 8,65 & $\mathbf{0 , 0 0 0 1}$ \\
\hline RESIDUAL & 15,9709 & 74 & 0,2158 & & \\
\hline TOTAL (CORRECTED) & 31,4108 & 79 & & & \\
\hline
\end{tabular}

All the F-ratios are based on the residual mean square error

\section{$>$ Multiple Range Test for MSE in the perturbation cases}

Table 9. (A) Method of 95,0\% LSD for MSE of control.

\begin{tabular}{|c|c|c|c|c|}
\hline CONTROL & Cases & Mean LS & $\sum$ LS & $\begin{array}{c}\text { Homogeneous } \\
\text { Groups }\end{array}$ \\
\hline Adapt FLC & 40 & 0,93575 & 0,073454 & $\mathbf{X}$ \\
\hline FLC & 40 & 1,62975 & 0,073454 & $\mathrm{X}$ \\
\hline
\end{tabular}

Table 9. (B)

\begin{tabular}{|l|c|c|c|}
\hline Contrast & Sig. & Difference & +/- Limits \\
\hline Adapt FLC - FLC & $*$ & $-0,694$ & 0,206987 \\
\hline \multicolumn{4}{|c}{$*$ Indicates a significant difference. }
\end{tabular}


The criterion of comparison between the conventional FLC and the proposed Adaptive and Self-organize FLC is the committed error (MSE) during the control process. Table 7 presents the results of all simulations performed during this study giving the MSE obtained of each algorithm using two sets of set-points.

In these cases of perturbations, we note that from the ANOVA Table 8 that the effects C: NOISE and B: CONTROL have a statistically significant effect on the MSE registered with a 95\% confidence level (both Pvalue $<0.05$ ), i.e. if we repeat the simulations with the same degree of perturbations there is a $95 \%$ risk of having the MSE in the same range, On the other hand, Table 9 (A and B) of multiple range test of the MSE for CONTROL prove that there is a statistically significant difference (between the MSE registered by our adaptive algorithm and the other algorithms in cases of disturbances) of $95 \%$ level of confidence, i.e. if we repeat the simulations several times, there is a $95 \%$ risk of having the same difference of error between our algorithm and the FLC.

\subsection{Conclusion}

This contribution presents a new adaptive fuzzy control policy that can guarantee a quite interesting reduction of the power consumed during a control process, the precision and the robustness of this control process has been widely represented and proved too. Our new policy has been able to carry out the control process with a very high performance against several conventional controllers in the same control process and under the same conditions. Noting that the adaptive controller designed and implemented in this paper can start the control of a quasi unknown plant, the only information needed is the delay and the monotonicity of the plant, also, the adaptive controller doesn't need any offline pre-training and can act without assigning any rule base and can learn from the real behavior of the plant in order to collect the required information to adapt the rules consequents (in real time) and to reorganize the membership functions. The validity and the extrapolability of the conclusions extracted from all obtained results were verified by a statistical analysis of variance (ANOVA).

\section{References}

1. R. Lasri, I. Rojas, H. Pomares and O. Valenzuela, "A New Adaptive Fuzzy Control Policy Against Conventional Methods. Statistical Analysis of Real Time Control Performance." 2012 International Conference on Complex Systems (ICCS), November, 5-6, 2012. Agadir, Morocco.

2. X. Wang, A. A. Stoorvogel, A. Saberi and P. Sannuti, "Discrete-time H-2 and H-infinity low-gain theory," Int. J. Robust. Nonlin, vol. 22, issue. 7, pp. 743-762, May. 2012.

3. Y. M. Li and Y. J. Du, "Indirect adaptive fuzzy observer and controller design based on interval type2 T-S fuzzy model," Appl. Math. Model, vol. 36, issue. 4, pp. 1558-1569, Apr. 2012.

4. E. H. Mamdani and S. Assilian, "Experiment in linguistic synthesis with a Fuzzy Logic Controller," Int.J.Man-Mach.Stud, vol. 7, pp. 1-13, 1975.

5. I. Rojas, H. Pomares, J. González, L. J. Herrera, A. Guillen, F. Rojas, O. Valenzuela, "Adaptive fuzzy controller: Application to the control of the temperature of a dynamic room in real time," Fuzzy Sets Syst., vol. 157, pp. 2241-2258, 2006.

6. I. Rojas, H. Pomares, J. González, L. J. Herrera, A. Guillen, F. Rojas, O. Valenzuela, "Adaptive fuzzy controller: Application to the control of the temperature of a dynamic room in real time," Fuzzy Sets Syst., vol. 157, pp. 2241-2258, 2006

7. T. Procyk and E. Mamdani, "A linguistic selforganizing process controller", Automatica, vol. 15, no. 1 , pp. 15-30, 1979.

8. C. F. Hsu, "Intelligent tracking control of a DC motor driver using self-organizing TSK-type fuzzy neural networks," Nonlinear Dynamics, vol. 67, issue. 1, pp. 587-600, Jan. 2012

9. C. S. Chen, "Robust Self-Organizing Neural-Fuzzy Control With Uncertainty Observer for MIMO Nonlinear Systems," IEEE Trans. Fuzzy Syst., vol. 19, issue. 4, pp. 694-706, Aug. 2011.

10. C. W. Park and M. Park, "Adaptive parameter estimator based on T-S fuzzy models and its applications to indirect adaptive fuzzy control design," Information Sciences, vol. 159, issues. 1-2, pp. 125139, 2004

11. H. Pomares, I. Rojas, J. González, F. Rojas, M. Damas and F. J. Fernández, "A two-stage approach to selflearning direct fuzzy controllers," Internat. J. Approx. Reason, vol. 29, issue. 3, pp. 267-289, 2002.

12. Y. W. Cho, C. W. Park and M. Park, "An indirect model reference adaptive fuzzy control for SISO Takagi-Sugeno model," Fuzzy Sets Syst., vol. 131, issue. 2, pp. 197-215, 2002. 
13. Y. W. Bai and C. H. Cheng, "Using an embedded controller with Fuzzy Logic to reduce power consumption of mobile computers". 36th Annual Conference on IEEE Industrial Electronics Society, pp. 236, IECON 2010.

14. C. B. Chiou, C. H. Chiou, C. M. Chu and S. L. Lin, "The application of fuzzy control on energy saving for multi-unit room airconditioners," Appl. Therm. Eng., vol. 29, issues. 2-3, pp. 310-316, 2009.

15. X. Shuo, Z. Ke, L. Zhe and Y. Fan. "Study of Fuzzy PID Control for a Semi-Track Air-Cushion Vehicle based on power consumption optimization," IEEE International Conference on Vehicular Electronics and Safety. On pp. 440, 2006.

16. R. Talebi-Daryani and J. Pfaff, "Intelligent control and power management of air conditioning systems using fuzzy logic and local operation networks," Automation Congress, Proceedings of the 5th Biannual World, vol. 14, pp. 195, 2002.

17. E. Kaymaz, "Adaptive environmental control for optimal energy consumption in hospitals," Proceedings of the Eighth IEEE Symposium on Computer-Based Medical Systems, pp. 165-172, Jun. 1995.

18. J. G. Ziegler and N. B. Nichols, "Optimum Settings for Automatic Controllers," Trans. ASME., vol. 64, pp. 759-768, 1942.

19. H. Andersen, A. Lotfi, and A. Tsoi, "A new approach to adaptive fuzzy control: the controller output error method," IEEE Trans. Syst., Man, Cybern. B, Cybern., vol. 27, no. 4, pp. 686-691, Aug. 1997.

20. P. A. Phan and T. J. Gale, "Direct adaptive fuzzy control with a self-structuring algorithm," Fuzzy Sets Syst., vol. 159, no. 8, pp. 871-899, 2008.

21. W. Wang, Y. Chien, and I. Li, "An on-line robust and adaptive T-S fuzzyneural controller for more general unknown systems," Int. J. Fuzzy Syst., vol. 10, no. 1, pp. 33-43, 2008.

22. M.Mucientes and J.Casillas, "Quick design of fuzzy controllerswith good interpretability in mobile robotics," IEEE Trans. Fuzzy Syst., vol. 15, no. 4, pp. 636-651, Aug. 2007.

23. Y. Liu andY. Zheng, "Adaptive robust fuzzy control for a class of uncertain chaotic systems," Nonlinear Dyn., vol. 57, no. 3, pp. 431-439, 2009.

24. C. T. Lin, C. J. Lin, and C. S. G. Lee, "Fuzzy adaptive learning control network with on-line neural learning," Fuzzy Sets Syst., vol. 71, no. 1, pp. 25-45, 1995. 